\title{
Digital Mechanism and Gear Library - Multimedia Collection of Text, Pictures and Physical Models
}

\author{
Rike Brecht, Torsten Brix, Ulf Döring, Veit Henkel, Heidi Krömker, \\ and Michael Reeßing \\ University of Technology Ilmenau, Germany \\ \{r.brecht, torsten.brix, ulf.doering, veit.henkel, heidi.kroemker, \\ michael.reessing\}@tu-ilmenau.de
}

\begin{abstract}
We are presenting a digital engineering library - the Digital Mechanism and Gear Library (DMG-Lib). The existing worldwide knowledge in form of books, drawings, physical models etc. is mostly scattered, difficult to access and does not comply with today's requirements concerning a rapid information retrieval. Therefore the development of a digital, internet-based library for mechanisms and gears is necessary, which makes the worldwide knowledge about mechanisms and gears available: http://www.dmg-lib.org. The Digital Mechanism and Gear Library is of particular importance not only for engineers, product designers and researchers, but also for teachers, students and historians.
\end{abstract}

Keywords: Multimedia, interactive collection, digital engineering library.

\section{Introduction}

The Digital Mechanism and Gear Library is a heterogeneous digital library with regard to the resources and media types. More than 3800 text documents, 1200 descriptions of mechanisms and machines, 540 videos and animations and 180 biographies of people in the domain of mechanism and machine science are available in the DMGLib in January 2009 and the collection is still growing. The multimedia collection consists of e-books, pictures, videos and animations.

\section{Physical Models}

The DMG-Lib concept and workflow takes into account that technical knowledge exists in different forms e.g. texts, pictures and physical models (see Figure1) and requires analytical, graphical and physical forms of representation.
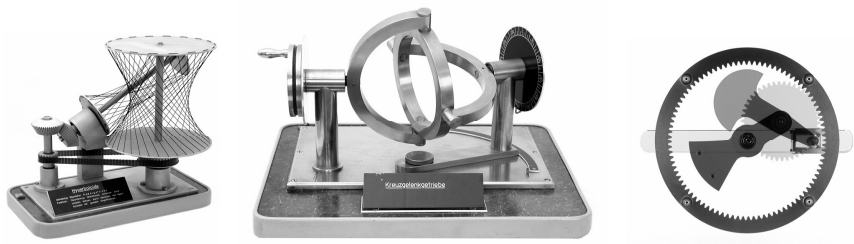

Fig. 1. Physical models digitized for DMG-Lib 
Therefore the focus is not only on textual documents and pictures. Thousands of unique physical models made of wood, Plexiglas or metal still exist illustrating and visualizing kinematic basics and methods. These functional models are digitized and available as videos and interactive animations.

\section{Interactive e-Books with Animation}

Typically text documents in the field of mechanisms and machine science are containing many figures. To better understand the motion of figured mechanisms and machines DMG-Lib can animate selected figures within e-books. These animations are augmenting original figures and can be displayed in the e-book pages with an integrated Java Applet.

http://www.dmg-lib.org/dmglib/main/portal.jsp?mainNaviState=browsen.docum

\section{DMG-Lib History Map}

The collection of DMG-Lib contains several items described with time and location based metadata. Persons' datasets contain metadata like place and date of birth as well as the other events of persons' CVs e.g. their appointment to university. Mechanism descriptions contain information on date of manufacturing or the actual abode. Metadata belonging to text documents are publication date and place. These metadata are presented in several maps using Google maps application interface. For example, DMG-Lib History Map illustrates the CVs of persons in the 1950s or shows the locations of all mechanism collections in Germany. The map can also be used to locate search results within the map in addition to a result list.

\section{Acknowledgments}

The DMG-Lib project (funded by DFG, German Research Foundation) started in 2003 with partners from TU Ilmenau, RWTH Aachen and TU Dresden. Further partners could be enlisted, e.g. the German committee of IFToMM. 